\title{
Compositional evolution of the emplaced fuel source in the vadose zone field experiment at Airbase Værløse, Denmark
}

Mette M. Broholm ${ }^{1}$, Mette Christophersen ${ }^{1}$, Uli Maier ${ }^{2}$, Erling H. Stenby ${ }^{3}$, Patrick Höhener ${ }^{4}$, and Peter Kjeldsen ${ }^{1}$.

${ }^{1}$ : Technical University of Denmark, Institute of Environment and Resources DTU, Bygningstorvet B115, DTU, DK-2800 Kgs. Lyngby, Denmark; e-mail mmb@er.dtu.dk

${ }^{2}$ : University of Tübingen, Center for Applied Geoscience, Sigwartstrasse 10, D-72076 Tübingen, Germany

3: Technical University of Denmark, IVC-SEP, Department of Chemical Engineering, Søltofts Plads, DTU, DK-2800 Kgs. Lyngby, Denmark

${ }^{4}$ : Laboratoire de Chimie et Environnement, Université de Provence, Case 29, 3, Place Victor Hugo, F13331 Marseille Cedex 3, France.

\section{Supporting information}


Chemical and physical parameters for the hydrocarbons selected for the source.

\begin{tabular}{|c|c|c|c|c|c|c|c|c|}
\hline & Compound & $\begin{array}{l}\text { Density } \\
{\left[\mathrm{g} \mathrm{mL}^{-1}\right]}\end{array}$ & $\begin{array}{c}\text { Molecular } \\
\text { Weight } \\
{\left[\mathrm{g} \mathrm{mol}^{-1}\right]}\end{array}$ & $\begin{array}{c}\text { Boiling } \\
\text { Point }\left[{ }^{0} \mathrm{C}\right]\end{array}$ & $\begin{array}{c}\text { Vapour } \\
\text { Pressure of } \\
\text { the pure } \\
\text { compound } \\
25^{0} \mathrm{C} \text { [kPa] }\end{array}$ & $\begin{array}{l}H[-] \\
25^{0} \mathrm{C}\end{array}$ & $\begin{array}{l}\text { Log } \\
\mathbf{K}_{\text {ow }}\end{array}$ & $\begin{array}{c}\text { Water } \\
\text { solubi- } \\
\text { lity } \\
{[\mathrm{mg} / \mathrm{l}]} \\
25^{\circ} \mathrm{C}\end{array}$ \\
\hline \multirow{4}{*}{ 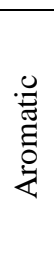 } & Benzene & 0.8765 & 78.11 & 80.1 & $12.672^{5}$ & $0.217^{3}$ & $2.13^{1}$ & $1790^{6}$ \\
\hline & Toluene & 0.8667 & $92.14^{2}$ & 110.63 & $3.805^{5}$ & $0.244^{3}$ & $2.73^{1}$ & $556^{5}$ \\
\hline & m-Xylene & 0.864 & 106.17 & 139.1 & $1.106^{5}$ & $0.26^{7}$ & $3.20^{1}$ & $158^{6}$ \\
\hline & $\begin{array}{c}1,2,4- \\
\text { Trimethyl- } \\
\text { benzene }\end{array}$ & 0.8761 & 120.19 & 169.3 & $0.271^{5}$ & $0.28^{8}$ & $3.78^{1}$ & $57^{4}$ \\
\hline \multirow{4}{*}{ 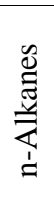 } & Hexane & 0.660 & 86.17 & 69.0 & $20.20^{9}$ & $68.58^{9}$ & $4.11^{14}$ & $9.5^{14}$ \\
\hline & Octane & 0.703 & 114.23 & 126.0 & $1.88^{9}$ & \multirow{3}{*}{$\begin{array}{l}121.0^{9} \\
197.85^{11} \\
296.77^{11}\end{array}$} & $5.15^{14}$ & $0.66^{14}$ \\
\hline & Decane & 0.730 & 148.28 & 174.1 & $0.175^{9}$ & & $6.25^{14}$ & $0.052^{14}$ \\
\hline & Dodecane & 0.749 & 170.34 & 216.3 & $0.0157^{9}$ & & $7.24^{14}$ & $0.0037^{14}$ \\
\hline \multirow{3}{*}{ 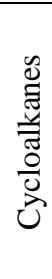 } & $\begin{array}{l}\text { Methyl- } \\
\text { cyclo- } \\
\text { pentane }\end{array}$ & 0.7486 & 84.16 & 71.8 & $18.4^{2,15}$ & $14.8^{15,18}$ & $3.37^{1,20}$ & $42^{21}$ \\
\hline & $\begin{array}{l}\text { Cyclo- } \\
\text { pentane }\end{array}$ & 0.7457 & 70.13 & 49.2 & $42.4^{16}$ & $7.6^{4}$ & $3.00^{1,20}$ & $156^{21,22}$ \\
\hline & $\begin{array}{l}\text { Methyl- } \\
\text { cyclohexane }\end{array}$ & 0.7694 & 98.19 & 100.9 & $6.13^{16}$ & $17.6^{19}$ & $3.61^{1,20}$ & $14^{21,22}$ \\
\hline \multirow{2}{*}{ 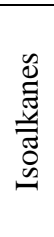 } & $\begin{array}{c}\text { 2,2,4- } \\
\text { Trimethyl- } \\
\text { pentane } \\
=\text { Isooctane }\end{array}$ & 0.692 & 114.2 & 99.2 & $6.56^{14}$ & $123.6^{14}$ & $4.09^{28}$ & $2.44^{14}$ \\
\hline & $\begin{array}{l}\text { 3-Methyl- } \\
\text { pentane }\end{array}$ & 0.664 & 84.16 & 64 & $25.3^{14}$ & $68.6^{14}$ & $3.60^{14}$ & $12.8^{14}$ \\
\hline 苋 & $\begin{array}{c}1,1,2- \\
\text { Trichloro- } \\
1,2,2- \\
\text { trifluoro- } \\
\text { ethane } \\
=\text { CFC-113 }\end{array}$ & 1.575 & 187.38 & 47.6 & $44.67^{23}$ & $14.20^{27}$ & $3.16^{8}$ & $170^{29}$ \\
\hline
\end{tabular}

${ }^{1}$ Hansch et al. (1995); ${ }^{2}$ US-EPA (1994); ${ }^{3}$ Peng and Wan $(1997) ;{ }^{4}$ Verschueren (1983); ${ }^{5}$ Shiu and Ma (2000); ${ }^{6}$ Montgomery and Welcom (1990); ${ }^{7}$ Dewulf et al. $(1995) ;{ }^{8}$ Hansen et al. (1995); $;{ }^{9}$ Mackay and Shiu (1981); ${ }^{10}$ Dean (1985); ${ }^{11}$ Yaws and Yang (1992); ${ }^{12}$ EPA (2000); ${ }^{13}$ Staudinger and Roberts (2001); ${ }^{14}$ Mackay et al. (1993); ${ }^{15}$ Boublik et al. (1984); ${ }^{16}$ Daubert and Danner (1989); ${ }^{17}$ Lide (1992); ${ }^{18}$ VP/WSOL - SRC PhysProb Database; ${ }^{19}$ Hine \& Mookerjee (1975); ${ }^{20}$ Biobyte Corp. (1994); ${ }^{21}$ McAuliffe (1966); ${ }^{22}$ Yalkowsky and Dannenfelser (1992); ${ }^{23}$ Yaws (1999); ${ }^{24}$ Milazzo (1956); ${ }^{25}$ Williamham (1945); ${ }^{26}$ Downing (1988); ${ }^{27} \mathrm{Bu}$ and Warner (1995); ${ }^{28}$ chemfinder.com; ${ }^{29}$ Horvath et al. (1999) 
Chemical and physical parameters for the hydrocarbons selected for the source (continued).

\begin{tabular}{|c|c|c|}
\hline & Compound & $\begin{array}{c}\text { Vapour pressure (pure compound) } \\
\text { Temperature dependency }\end{array}$ \\
\hline 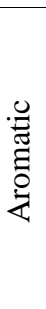 & $\begin{array}{l}\text { Benzene } \\
\text { Toluene } \\
\text { m-Xylene } \\
\begin{array}{l}1,2,4 \text { Trimethyl- } \\
\text { benzene }\end{array}\end{array}$ & $\begin{aligned} & \text { For } \mathbf{t}=\mathbf{5 . 0 - 5 0} \mathbf{C}^{\mathbf{5}} \\
\log (\mathrm{p}[\mathrm{kPa}])= & 6.02994-1211.033 /(\mathrm{T}[\mathrm{K}]-52.36) \\
& \text { For } \mathbf{t}=\mathbf{5 . 0 - 5 0} \mathbf{C}^{\mathbf{5}} \\
\log (\mathrm{p}[\mathrm{kPa}])= & 6.02994-1391.005 /(\mathrm{T}[\mathrm{K}]-48.974) \\
& \text { For } \mathbf{t}=\mathbf{5 . 0 - 5 0} \mathbf{C}^{\mathbf{5}} \\
\log (\mathrm{p}[\mathrm{kPa}])= & 6.134008-1462.266 /(\mathrm{T}[\mathrm{K}]-58.045) \\
& \text { For } \mathbf{t}=\mathbf{5 . 0 - 5 0} \mathbf{C}^{\mathbf{5}} \\
\log (\mathrm{p}[\mathrm{kPa}])= & 6.16866-1573.267 /(\mathrm{T}[\mathrm{K}]-64.586)\end{aligned}$ \\
\hline 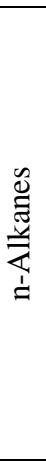 & $\begin{array}{l}\text { Octane } \\
\text { Decane } \\
\text { Dodecane }\end{array}$ & $\begin{array}{c}\text { For } \mathbf{t}=\mathbf{- 2 5 - 9 2} \mathbf{C}^{\mathbf{1 0}} \\
\log \mathrm{p}[\mathrm{mmHg}]=\mathrm{A}-\mathrm{B} /\left(\mathrm{t}\left[{ }^{\circ} \mathrm{C}\right]+\mathrm{C}\right) \\
\mathrm{A}=6.87601 ; \mathrm{B}=1171.17 ; \mathrm{C}=224.41 \\
\text { For } \mathbf{t}=\mathbf{1 9 - 1 5 2} \mathbf{C}^{\mathbf{1 0}} \\
\log \mathrm{p}[\mathrm{mmHg}]=\mathrm{A}-\mathrm{B} /\left(\mathrm{t}\left[{ }^{\circ} \mathrm{C}\right]+\mathrm{C}\right) \\
\mathrm{A}=6.91868 ; \mathrm{B}=1351.99 ; \mathrm{C}=209.15 \\
\text { For } \mathbf{t}=\mathbf{5 8 - 2 0 3} \mathbf{C}^{\mathbf{1 0}} \\
\left.\log \mathrm{p}[\mathrm{mmHg}]=\mathrm{A}-\mathrm{B} /\left(\mathrm{t}^{\circ} \mathrm{C}\right]+\mathrm{C}\right) \\
\mathrm{A}=6.94365 ; \mathrm{B}=1495.17 ; \mathrm{C}=193.86 \\
\text { For } \mathbf{t}=\mathbf{9 1 - 2 4 7} \mathbf{C}^{\mathbf{1 0}} \\
\log \mathrm{p}[\mathrm{mmHg}]=\mathrm{A}-\mathrm{B} /\left(\mathrm{t}\left[{ }^{\circ} \mathrm{C}\right]+\mathrm{C}\right) \\
\mathrm{A}=6.99795 ; \mathrm{B}=1639.27 ; \mathrm{C}=181.84\end{array}$ \\
\hline 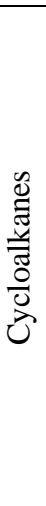 & $\begin{array}{l}\text { Cyclo-pentane } \\
\text { Methyl- } \\
\text { cyclohexane }\end{array}$ & $\begin{array}{c}\text { For } \mathbf{t}=\mathbf{- 0 . 6 - 1 7 . 9} \mathbf{C}^{17}: \mathrm{P}^{0}=0.432 \mathrm{t}+5.59 \\
\text { For } \mathbf{t}=\mathbf{- 4 7 . 3 - 1 4 . 2} \mathbf{C}^{\circ} \mathbf{C}^{15}: \\
\log \mathrm{p}[\mathrm{kPa}]=\mathrm{A}-\mathrm{B} /\left(\mathrm{t}\left[{ }^{\circ} \mathrm{C}\right]+\mathrm{C}\right) \\
\mathrm{A}=6.25832 ; \mathrm{B}=1240.438 ; \mathrm{C}=242.957 \\
\text { For } \mathbf{t}=\mathbf{1 5 . 7 - 5 0 . 0} \mathbf{C}^{15}: \\
\log \mathrm{p}[\mathrm{kPa}]=\mathrm{A}-\mathrm{B} /\left(\mathrm{t}\left[{ }^{\circ} \mathrm{C}\right]+\mathrm{C}\right) \\
\mathrm{A}=6.02877 ; \mathrm{B}=1133.199 ; \mathrm{C}=232.415 \\
\text { For } \mathbf{t}=\mathbf{- 3 . 2 - 2 2} \mathbf{C}^{17}: \\
\mathrm{P}^{0}=0.216 \mathrm{t}+1.46 \\
\text { For } \mathbf{t}=\mathbf{2 5 . 6 - 1 0 1} \mathbf{C}^{15}: \\
\log \mathrm{p}[\mathrm{kPa}]=\mathrm{A}-\mathrm{B} /\left(\mathrm{t}\left[{ }^{\circ} \mathrm{C}\right]+\mathrm{C}\right) \\
\mathrm{A}=5.95366 ; \mathrm{B}=1273.962 ; \mathrm{C}=221.755\end{array}$ \\
\hline 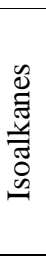 & $\begin{array}{l}\text { 2,2,4-Trimethyl- } \\
\text { pentane } \\
=\text { Isooctane } \\
\text { 3-Methyl- } \\
\text { pentane }\end{array}$ & 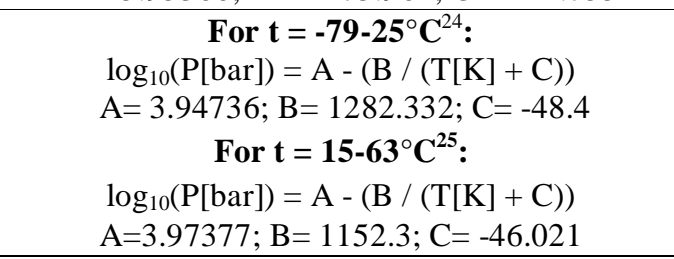 \\
\hline 泀 & $\begin{array}{c}\text { 1,1,2-Trichloro- } \\
\text { 1,2,2- trifluoro- } \\
\text { ethane } \\
=\text { CFC- } 113\end{array}$ & $\begin{array}{l}\mathrm{P} 0\left(25^{\circ} \mathrm{C}\right): 44.67 \mathrm{kPa}^{26} \\
\mathrm{P} 0\left(20^{\circ} \mathrm{C}\right): 36.31 \mathrm{kPa}^{26}\end{array}$ \\
\hline
\end{tabular}




\section{$\underline{\text { Source NAPL saturation }}$}

In unsaturated soils the larger pores are filled with the gas-phase, water covers the surfaces of the soil grains and fills the smallest pores, and NAPL is present along the water-gas interface $(30,31)$. Average residual saturations (= retention capacities) for two NAPL (PCE) releases to the vadose zone sand were $2.8 \%$ of the pore-volume and $1.0 \%$ of the pore-volume, respectively, with individual sample residual saturations of up to $19.6 \%$ of the pore-volume (32). The water content was on the order of $16 \%$ of the pore-volume. Jarsjö et al. (33) found a residual saturation of $10 \%$ of the porevolume for kerosine in sand at a water content of $17 \%$ of the pore-volume in laboratory column experiments.

Emplacement of sand-NAPL mixtures with varying NAPL saturation $(7.5 \%, 10 \%$, $12.5 \%, 15.2 \%, 20 \%)$ on clean naturally water-wet sand from the field site was tested in the laboratory. No remobilisation occurred for NAPL saturations of 7.5\%-12.5\% of the pore-volume. Remobilisation occurred for $20 \%$ NAPL saturation, whereas only very little NAPL remobilised into the underlying sand for a residual concentration of $15.2 \%$.

Various NAPL saturations, NAPL volume and source volume compositions, were evaluated prior to the installation of the source in order to assure a greater than 1 year duration of source. The details and results are given in (34). Based on this and the laboratory tests, it was decided to aim for a NAPL saturation of $12.5 \%$ in the field experiment. The MINTEQ model (see main paper and below) was then used to evaluate the depletion of the individual compounds in the source after 1 year for the planned composition for a constant temperature of $10^{\circ} \mathrm{C}$ and neglecting degradation of the compounds. The model predicted the depletion of the 4 most volatile compounds within 1 year (data not shown). 
Pore-air concentrations (mg/l) calculated from source composition based on Raoult's Law and on the modified Raoult's Law compared to measured pore-air concentrations in the source.

\begin{tabular}{|c|c|c|c|c|c|c|c|c|c|c|c|}
\hline 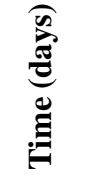 & 芑 & 己े & 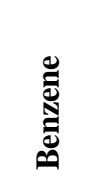 & 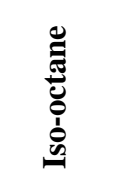 & $\underline{\underline{z}}$ & & 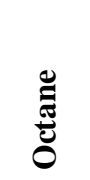 & 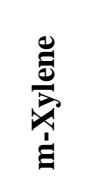 & $\sum_{i}^{\bullet}$ & 芯 & 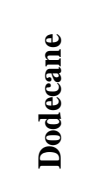 \\
\hline 0 & RL & $\begin{array}{c}36,5 \pm \\
2,07\end{array}$ & $\begin{array}{c}4,44 \pm \\
0,31\end{array}$ & $\begin{array}{c}34,1 \pm \\
1,12\end{array}$ & $\begin{array}{c}21,5 \pm \\
0,30\end{array}$ & $\begin{array}{c}2,69 \pm \\
0,06\end{array}$ & $\begin{array}{c}4,40 \pm \\
0,15\end{array}$ & $\begin{array}{c}1,61 \pm \\
0,04\end{array}$ & $\begin{array}{c}0,89 \pm \\
0,03\end{array}$ & $\begin{array}{c}0,84 \pm \\
0,04\end{array}$ & $\begin{array}{l}0,04 \pm \\
0,003\end{array}$ \\
\hline 0 & MRL & 35,4 & 5,72 & 34,4 & 21,5 & 3,60 & 4,45 & 1,88 & 1,01 & 0,81 & 0,04 \\
\hline $0-13$ & MS & $\sim 41 \downarrow$ & $\begin{array}{r}3,5- \\
5,6 \downarrow\end{array}$ & $\begin{array}{c}23,4- \\
35,9\end{array}$ & $\begin{array}{l}13,9- \\
20,7\end{array}$ & $\begin{array}{c}3,4- \\
4,6\end{array}$ & $\begin{array}{l}1,8- \\
2,9\end{array}$ & $\begin{array}{c}0,8- \\
1,2\end{array}$ & $\begin{array}{c}0,4- \\
0,6\end{array}$ & $\begin{array}{c}0,2- \\
0,3\end{array}$ & $\tilde{0,001}$ \\
\hline 23 & RL & $\begin{array}{c}10,5 \pm \\
1,86\end{array}$ & $\begin{array}{c}0,46 \pm \\
0,13\end{array}$ & $\begin{array}{c}28,8 \pm \\
2,96\end{array}$ & $\begin{array}{c}19,2 \pm \\
1,47\end{array}$ & $\begin{array}{c}2,66 \pm \\
0,14\end{array}$ & $\begin{array}{c}5,79 \pm \\
0,17\end{array}$ & $\begin{array}{c}2,14 \pm \\
0,08\end{array}$ & $\begin{array}{c}1,30 \pm \\
0,08\end{array}$ & $\begin{array}{c}1,14 \pm \\
0,06\end{array}$ & $\begin{array}{l}0,05 \pm \\
0,003\end{array}$ \\
\hline 23 & MRL & 9,94 & 0,56 & 29,37 & 18,96 & 3,38 & 5,91 & 2,42 & 1,44 & 1,14 & 0,05 \\
\hline $\begin{array}{l}17- \\
37\end{array}$ & MS & $\begin{array}{l}13,2- \\
28,7 \downarrow\end{array}$ & $\begin{array}{l}1,2- \\
2,5 \downarrow\end{array}$ & $\begin{array}{l}22,2- \\
29,8\end{array}$ & $\begin{array}{c}13,6- \\
17,5\end{array}$ & $\begin{array}{c}3,0- \\
4,1\end{array}$ & $\begin{array}{l}1,3- \\
2,6\end{array}$ & $\begin{array}{c}0,40- \\
0,94\end{array}$ & $\begin{array}{c}0,24- \\
0,57\end{array}$ & $\begin{array}{c}0,19- \\
0,34\end{array}$ & $\begin{array}{c}0,004 \\
0,02\end{array}$ \\
\hline 113 & RL & 0 & $\begin{array}{l}0,02 \pm \\
0,003\end{array}$ & $\begin{array}{c}7,62 \pm \\
0,66 \\
\end{array}$ & $\begin{array}{c}4,61 \pm \\
0,37 \\
\end{array}$ & $\begin{array}{c}0,57 \pm \\
0,05\end{array}$ & $\begin{array}{c}4,20 \pm \\
0,35 \\
\end{array}$ & $\begin{array}{c}1,74 \pm \\
0,12 \\
\end{array}$ & $\begin{array}{l}1,41 \pm \\
0,05\end{array}$ & $\begin{array}{c}1,30 \pm \\
0,06\end{array}$ & $\begin{array}{l}0,06 \pm \\
0,005 \\
\end{array}$ \\
\hline 113 & MRL & 0 & 0,02 & 7,78 & 4,48 & 0,69 & 4,29 & 1,93 & 1,54 & 1,33 & 0,05 \\
\hline $\begin{array}{l}100- \\
125\end{array}$ & MS & $\begin{array}{l}0,01- \\
0,44 \downarrow\end{array}$ & $\begin{array}{c}0- \\
0,01 \downarrow\end{array}$ & $\begin{array}{c}7,9- \\
11,2 \downarrow\end{array}$ & $\begin{array}{l}4,2- \\
5,7 \downarrow\end{array}$ & $\begin{array}{c}0,7- \\
2,5 \downarrow\end{array}$ & $\begin{array}{l}0,7- \\
5,1 \downarrow\end{array}$ & $\begin{array}{c}0,33- \\
2,1\end{array}$ & $\begin{array}{l}0,2- \\
1,13\end{array}$ & $\begin{array}{c}0,07- \\
0,65\end{array}$ & $0-0,01$ \\
\hline 225 & RL & 0 & 0 & $\begin{array}{c}0,80 \pm \\
0,20\end{array}$ & $\begin{array}{c}0,48 \pm \\
0,09\end{array}$ & $\begin{array}{l}0,03 \pm \\
0,005\end{array}$ & $\begin{array}{c}1,90 \pm \\
0,11\end{array}$ & $\begin{array}{c}0,91 \pm \\
0,06\end{array}$ & $\begin{array}{c}0,99 \pm \\
0,02\end{array}$ & $\begin{array}{c}0,92 \pm \\
0,03\end{array}$ & $\begin{array}{l}0,03 \pm \\
0,001\end{array}$ \\
\hline 225 & MRL & 0 & 0 & 0,81 & 0,46 & 0,04 & 1,92 & 1,00 & 1,08 & 0,93 & 0,03 \\
\hline $\begin{array}{l}205- \\
237\end{array}$ & MS & $\begin{array}{l}0,0003- \\
0,004 \downarrow\end{array}$ & 0 & $\begin{array}{l}0,69- \\
1,9 \downarrow\end{array}$ & $\begin{array}{l}0,16- \\
1,0 \downarrow\end{array}$ & $\begin{array}{c}0,05- \\
0,16\end{array}$ & $\begin{array}{c}0,75- \\
1,6\end{array}$ & $\begin{array}{l}0,25- \\
0,76\end{array}$ & $\begin{array}{c}0,14- \\
0,48\end{array}$ & $\begin{array}{c}0,13- \\
0,35\end{array}$ & $\begin{array}{c}0- \\
0,004\end{array}$ \\
\hline 352 & RL & 0 & 0 & $\begin{array}{c}0,005 \pm \\
0,007\end{array}$ & $\begin{array}{c}0,02 \pm \\
0,01\end{array}$ & 0 & $\begin{array}{c}1,07 \pm \\
0,30 \\
\end{array}$ & $\begin{array}{c}0,64 \pm \\
0,17 \\
\end{array}$ & $\begin{array}{c}1,97 \pm \\
0,17 \\
\end{array}$ & $\begin{array}{c}2,12 \pm \\
0,05\end{array}$ & $\begin{array}{c}0,11 \pm \\
0,01 \\
\end{array}$ \\
\hline 352 & MRL & 0 & 0 & 0,005 & 0,02 & 0 & 1,09 & 0,65 & 2,22 & 2,18 & 0,11 \\
\hline $\begin{array}{c}329- \\
350 \\
(364)\end{array}$ & MS & $\begin{array}{c}0- \\
0,0003\end{array}$ & 0 & $\begin{array}{l}0,11- \\
0,33 \downarrow\end{array}$ & $\begin{array}{l}0,04- \\
0,15 \downarrow\end{array}$ & $\begin{array}{c}0- \\
0,002 \downarrow\end{array}$ & $\begin{array}{l}0,84- \\
2,8 \downarrow\end{array}$ & $\begin{array}{l}0,5- \\
2,5 \uparrow\end{array}$ & $\begin{array}{l}0,77- \\
6,9 \uparrow\end{array}$ & $\begin{array}{l}0,02- \\
4,89 \uparrow\end{array}$ & $\begin{array}{c}0,03- \\
0,92 \uparrow\end{array}$ \\
\hline
\end{tabular}

RL: Raoult's Law calculation, \pm standard deviation.

MRL: Modified Raoult's Law (with UNIFAC-predicted -values) calculation,

Meas.: Range of measured pore-air concentrations in the source (S-0-4, S-0-6, S-0-7) for the timeperiod, (low values likely biased by sampling have been left out).

$\downarrow$ : Decreasing trend, $\uparrow:$ Increasing trend. Decreasing trends for several compounds during the period $70-154$ days is primarily due to decreasing temperature.

(364): Day 364 only included for S-0-7. 
Modelling parameters for the variably saturated flow and reactive transport model using MIN3P (35) for the 3D simulation of the Værløse field experiment.

\section{Model geometry}

Cartesian coordinates

$\mathrm{x}$ - (GW flow) direction: length: $6.5 \mathrm{~m}$, number of cells: 17, spacing: $25 \mathrm{~cm}$ (around source), $40 \mathrm{~cm}$ (distant)

y-direction: $\quad$ length: $6.5 \mathrm{~m}$, number of cells: 17, spacing: $25 \mathrm{~cm}$ (around source), $40 \mathrm{~cm}$

(distant)

z- direction (vertical): depth: $4.0 \mathrm{~m}$, number of cells: 40 , spacing: $10 \mathrm{~cm}$

Top boundary (variably saturated flow): daily averaged precipitation or evapotranspiration, resp.

Top boundary (reactive transport): fixed concentration (atmospheric conditions)

Bottom boundary: impermeable (aquitard)

Inflow boundary (variably saturated flow): fixed flow $q=f($ time) to account for measured water table elevation

Inflow boundary (reactive transport): clean groundwater with oxygen saturation

Outflow boundary (variably saturated flow): fixed head $h=f($ time $)$ to account for measured water table elevation

Outflow boundary (reactive transport): zero gradient

Nodes outside source zone: 16 laterally (x, y), 30 vertically

Meteorological conditions:

Temperature ( $T$ ): time and depth dependent $T$ profiles, interpolated from daily measurements of air and source $T$ and frequently measured depth profiles in the vicinity of the source.

$\begin{array}{lr}\text { Maximum } T: & 22.8{ }^{\circ} \mathrm{C} \\ \text { Minimum } T: & -14.05{ }^{\circ} \mathrm{C} \\ \text { Average } T: & 10.24{ }^{\circ} \mathrm{C}\end{array}$

Precipitation $(P)$ : daily measured values from a weather station at the site

$$
\text { Maximum } P: \quad 25.8 \mathrm{~mm} / \text { day }
$$

Total $P$ (350 days): $\quad 654.8 \mathrm{~mm}$

Evapotranspiration (ET): potential evapotranspiration $E T_{P o t}$ according to Haude (33), corrected for actual evapotranspiration $E T_{A k t}$ using MIN3P
Maximum ET:
$5.0 \mathrm{~mm} /$ day
Total $E T_{\text {Pot }}$ (350 days):
$332.4 \mathrm{~mm}$
Total $E T_{A k t}$ (350 days):
$160.3 \mathrm{~mm}$

Total $P-E T_{A k t}$ (groundwater recharge within 350 days): $494.5 \mathrm{~mm}$

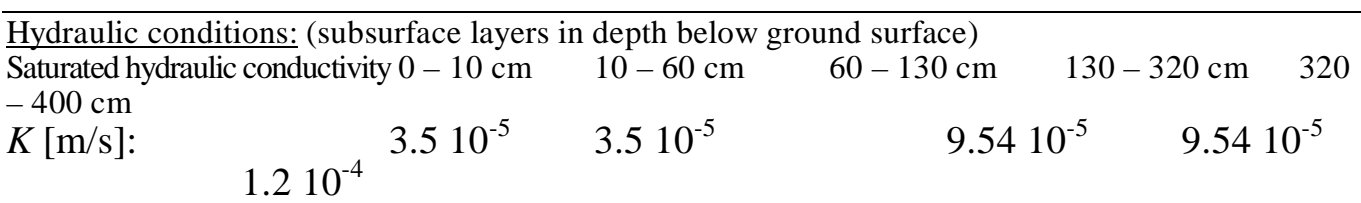

Groundwater flow velocity $v$ : between $0.206-0.278 \mathrm{~m}^{\text {day }}{ }^{-1}$

Water table: frequently measured elevations implemented, between 0.3 and $1.6 \mathrm{~m}$ above aquifer bottom

Source zone:

Symmetry boundary at the source centre in $\mathrm{x}$ - and y-direction (splitted in 1/4)

Volume: $0.27 \mathrm{~m}^{3} ; 10 \times 1$ grid elements (vertically, laterally, respectively), from $0.8-$

$1.8 \mathrm{~m}$ depth, taking downward migration of the NAPL into account

NAPL volume fraction: $3.75 \%$

Composition: 13 kerosene compounds, tracer CF113, according to Tab. 1

Porous medium 
Diffusion coefficients: aqueous $D_{a q}=8.0 \mathrm{E}-10 \mathrm{~m}^{2} / \mathrm{s} ;$ gaseous $D_{a i r}=7.3 \mathrm{E}-6 \mathrm{~m}^{2} / \mathrm{s}$

\begin{tabular}{|c|c|c|c|c|c|}
\hline $\begin{array}{l}\text { Subsurface layers (depth) } \\
-400 \mathrm{~cm}\end{array}$ & $0-10 \mathrm{~cm}$ & $10-60 \mathrm{~cm}$ & $60-130 \mathrm{~cm}$ & $130-320 \mathrm{~cm}$ & 320 \\
\hline $\begin{array}{l}\text { Porosity [-]: } \\
\%\end{array}$ & $40 \%$ & $39 \%$ & $34 \%$ & $35 \%$ & 35 \\
\hline Residual saturation $\theta_{\text {res }}$ : & $9 \%$ & $9 \%$ & $7.5 \%$ & $7.5 \%$ & $8 \%$ \\
\hline \multicolumn{6}{|c|}{ Van Genuchten parameters (determined by Lange (37)) } \\
\hline$\alpha\left[\mathrm{m}^{-1}\right]:$ & 2.9 & 2.9 & 3.2 & 3.2 & 3.2 \\
\hline$n:$ & 1.84 & 1.84 & 2.97 & 2.97 & 2.97 \\
\hline \multicolumn{6}{|l|}{ Transport parameters: } \\
\hline \multicolumn{6}{|l|}{ Dispersivity [m] } \\
\hline Longitudinal $\alpha_{L}$ & 0.01 & 0.01 & 0.01 & 0.1 & 0.1 \\
\hline Transverse horizontal $\alpha_{T H}$ & 0.001 & 0.001 & 0.001 & 0.02 & 0.02 \\
\hline Transverse vertical $\alpha_{T V}$ & 0.001 & 0.001 & 0.001 & 0.02 & 0.02 \\
\hline
\end{tabular}

Numerical parameters:

Time step: variable between $10^{-8}$ and 1 day (depending on gradients/convergence)

Spatial weighting: upstream

Solver residual tolerance: $10^{-8}$

Total elapsed time: 350 days (duration of the experiment) 
Capabilities of MIN3P that were used in the 3D field site model are given in the following table:

\begin{tabular}{|c|c|c|}
\hline $\begin{array}{ll}1.1 & \text { Capabilities of } \\
\text { MIN3P }\end{array}$ & $\begin{array}{l}\text { Implementation in } \\
\text { the 3D Værløse field } \\
\text { site model }\end{array}$ & Formulation \\
\hline \multirow[t]{2}{*}{$\begin{array}{l}\text { Phase partitioning } \\
\text { (aqueous - gaseous) }\end{array}$} & $\begin{array}{l}\begin{array}{l}\text { Henry's law } \\
\text { (equilibrium) }\end{array} \\
\end{array}$ & $C_{g}=C_{w} H$ \\
\hline & $\begin{array}{l}\text { Temperature } \\
\text { dependence (Van't } \\
\text { Hoff equation) }\end{array}$ & $\ln H_{T}=\ln H_{298.15 K}-\frac{\Delta H_{0}}{R}\left(\frac{1}{T}-\frac{1}{298.15 K}\right)$ \\
\hline $\begin{array}{l}\text { NAPL dissolution/ } \\
\text { volatilisation }\end{array}$ & $\begin{array}{l}\text { Raoults' law } \\
\text { (equilibrium) }\end{array}$ & $C_{\text {Sat }}^{R}=C_{\text {Sat }}^{W} \chi_{i}$ \\
\hline \multirow[t]{2}{*}{ Variably saturated flow } & Richards equation & $S_{a} S_{S} \frac{\partial h}{\partial t}+n \frac{\partial S_{a}}{\partial t}=\nabla \cdot\left(k_{r a} K \nabla h\right)+Q_{a}$ \\
\hline & $\begin{array}{l}\text { Van Genuchten } \\
\text { approach, using the } \\
\text { empirical factors } \\
\alpha\left[\mathrm{m}^{-1}\right] \\
n[-] \\
m=1-1 / n[-] \\
l[-]\end{array}$ & $\begin{array}{l}S_{a}=S_{r a}+\frac{1-S_{r a}}{\left(1+\alpha \psi_{a}^{n}\right)^{m}} \\
k_{r a}=S_{e a}^{l}\left[1-\left(1-S_{e a}^{1 / m}\right)^{m}\right]^{2} \\
S_{e a}=\frac{S_{a}-S_{r a}}{1-S_{r a}}\end{array}$ \\
\hline \multirow[t]{2}{*}{ Vapour phase diffusion } & Fick's second law & $\alpha_{g} \frac{\partial C_{g}}{\partial t}=-\nabla \cdot F=\nabla \cdot D_{e f f} \nabla C_{g}$ \\
\hline & $\begin{array}{l}\text { Millington } \\
\text { relationship for } \\
\text { tortuosity correction }\end{array}$ & $D_{e f f}=D_{\text {air }} \frac{n S_{g}}{\tau_{g}} ; \tau_{g}=S_{g}{ }^{7 / 3} n^{1 / 3}$ \\
\hline $\begin{array}{l}\text { Intra aqueous kinetic } \\
\text { reactions }\end{array}$ & $\begin{array}{l}\text { Used for aerobic } \\
\text { hydrocarbon } \\
\text { degradation (pseudo } \\
\text { first order) } \\
\end{array}$ & $R_{k}^{a}=-k_{k}^{a} \prod_{v<0}\left(\gamma_{i} C_{i}\right)^{-v}$ \\
\hline $\begin{array}{l}\text { Transient boundary } \\
\text { conditions }\end{array}$ & \multicolumn{2}{|c|}{$\begin{array}{l}\text { Used for variably saturated flow simulation, implementation of } \\
\text { precipitation, evapotranspiration and water table fluctuations }\end{array}$} \\
\hline Temperature profile & \multicolumn{2}{|c|}{$\begin{array}{l}\text { Depth and time dependent, used for implementation of measured and } \\
\text { interpolated values }\end{array}$} \\
\hline
\end{tabular}

\section{Notation:}

$\alpha_{g}=n S_{g}+n S_{w} H^{-1}+K_{d} \rho H^{-1} \quad$ mass capacity factor [-] with respect to the gaseous phase

$C_{g}$ : gaseous concentration $[\mathrm{mol} / \mathrm{l}]$

$C_{w}:$ aqueous concentration $[\mathrm{mol} / 1]$

$C_{{ }_{S a t}}^{R}$ : aqueous saturation concentration from mixture [mol/l]

$C^{W}{ }_{\text {Sat }}$ : aqueous saturation concentration for pure compound [mol/1]

$D_{\text {air }}$ : free air diffusion coefficient $\left[\mathrm{m}^{2} / \mathrm{s}\right]$

$D_{e f f}:$ effective diffusion coefficient for porous medium $\left[\mathrm{m}^{2} / \mathrm{s}\right]$

$F$ : diffusive mass flux $\left[\mathrm{mol} / \mathrm{m}^{2} /\right.$ day] in the gaseous phase

$\gamma_{i}$ : activity coefficient [-] for a specific compound I, (generally $=1$ )

$h$ : hydraulic head $[\mathrm{m}]$

$\psi_{a}$ : aqueous phase pressure head $\psi_{a}=h-z[-]$ (z: elevation)

$H$ : Henry's law constant [-], $H_{T}$ Henry's law constant at given temperature $T$

$\Delta H_{0}$ : stanard enthalpy of gas-aqueous phase partitioning for the compound [ $\left.\mathrm{kJ} \mathrm{mol}^{-1}\right]$

$k_{k}^{a}$ : aqueous phase reaction rate constant for reaction $\mathrm{k}$ [day $\left.{ }^{-1}\right]$, (pseudo-first order approach)

$k_{r a}$ : relative permeability of the porous medium with respect to the aqueous phase [-]

$K$ : hydraulic conductivity tensor

$n$ : total porosity [-]

$v$ : stoichiometric coefficient [-]

$Q_{a}:$ aqueous flow source/sink-term $\left[\mathrm{m}^{3} / \mathrm{s}\right]$

$\chi_{i}$ : mole fraction of a compound in a mixture [-]

$R$ : universal gas constant $\left(8.31434 \mathrm{~J} \mathrm{~mol}^{-1} \mathrm{~K}^{-1}\right)$ 
$R_{k}^{a}$ : aqueous phase reaction rate for reaction $\mathrm{k}$ [mol/1/day]

$S_{a}$ : aqueous phase saturation [-]

$S_{e a}:$ effective saturation of the aqueous phase [-]

$S_{g}$ : gas phase saturation [-]

$S_{r a}:$ residual saturation for the aqueous phase [-]

$S_{S}$ : specific storage coefficient $\left[\mathrm{m}^{-1}\right]$

$\tau_{g}$ : tortuosity factor [-] with respect to the gaseous phase

\section{Literature Cited}

(1) Hansch, C.; Leo, A.; Hoekman, D. Exploring QSAR - Hydrophobic, Electronic, and Steric Constants. Washington, DC: American Chemical Society, 1995.

(2) United States Environmental Protection Agency. Locating and Estimating Air Emissions from Sources of Toluene. EPA-454/R-93-047, 1994.

(3) Peng, J.; Wan, A. Measurement of Henry's constants of of high-volatility organic compounds using a headspace autosampler. Environ. Sci. Technol., 1997, 31, 2998-3003.

(4) Verschueren K (Ed). Handbook of Environmental Data on Organic Chemicals, 2nd ed., Van Nostrand Reinhold Co., New York. 1983, 1162-1163.

(5) Shiou, W.-Y.; Ma, K.-C. Temperature dependence of physical-chemical properties of selected chemicals of environmental interest. J. Phys. Chem. Ref. Data, 2000, 29(1), .

(6) Montgomery, J.H.; Welcom, L.M. Groundwater chemicals desk reference. Lewis publishers Inc, Michigan. 1990.

(7) Dewulf, J.; Drijvers, D.; van Langenhove, H. Measurements of Henry's law constants as a function of temperature and salinity for the low temperature range. Atmos. Environ., 1995, 29, 323-331.

(8) Hansen, K.C.; Zhou, Z.; Yaws, C.L.; Aminabhavi, T.M. A laboratory method for the determination of Henry's law constants of volatile organic chemicals. J. Chem. Educ., 1995, 72, 93-96.

(9) Mackay, D.; Shiu, W.Y. A critical review of Henry's law constants for chemicals of environmental interest. J. Phys. Chem. Ref. Data, 1981, 10, 1175-1199.

(10) Dean, J.A. Lange's Handbook of Chemistry, 13 th Edition, McGraw-Hill Book Company ISBN 0-07-016192-5, Table 10-8: Vapor Pressures of Various Organic Compounds,1985, pp. 10-37 to 10-54.

(11) Yaws, C.L.; Yang, H.-C. Henry's law constant for compound in water. In Thermodynamic and Physical Property Data, C. L. Yaws, ed(s)., Gulf Publishing Company, Houston, TX, 1992, 181-206.

EPA (2000), EPI SUITE - bond estimation method for H calculation 
(13) Staudinger, J.; Roberts, P.V. A critical compilation of Henry's law constant temperature dependence relations for organic compounds in dilute aqueous solutions. Chemosphere, 2001, 44, 561-576

(14) Mackay, D.; Shiu, W.Y.; Ma, K.C. Illustrated Handbook of physical-chemical properties and environmental fate for organic chemicals, Vol. 3. Volatile organic chemicals. 1993, Boca Raton: Lewis. 916.

(15) Boublik, T.; Fried, V.; Hála, E. The vapour pressures of pure substances, selected values of the temperature dependence of the vapour pressures of some pure substances in the normal and low pressure region. Physical sciences data 17, Elsevier, 1984, second revised edition, Amsterdam, The Netherlands.

(16) Daubert,T.E.; Danner, R.P. Physical and thermodynamic properties of pure chemicals: Data compilation, Design Institute for Physical Property Data, American Institute of Chemical Engineers. Hemisphere Pub. Corp., New York, 4 Vol., 1989.

(17) Lide, D.R. Handbook of chemistry and physics $1992-1993,73^{\text {rd }}$ ed., 1992, CRC Press, Boca Roaton, Florida, US.

(18) SRC PhysProp Database, http://esc.syrres.com/interkow/PhysProp.htm

(19) Hine, J.; Mookerjee, P.K. The intrinsic hydrophilic character of organic compounds. Correlations in terms of structural contributions. J. Org. Chem., 1975, 40, 292-298.

(20) Biobyte Corp., ClogP for Windows, 1.0.0, MedChem, 1994, Claremont, CA.

(21) McAuliffe, C. Solubility in water of paraffin, cycloparrafin, olefin, acetylene olefin and aromatic hydrocarbons. J. Phys. Chem., 1966, 70 (4), 1267-1275.

(22) Yalkowsky, S.H.; Dannenfelser, R.M. Aquasol database of aqueous solubility, Version 5, PC Version, College of Pharmacy, Univ. of Arizona - Tucson, AZ.

(23) Yaws, C.L. Chemical properties handbook: physical, thermodynamic, environmental and inorganic chemicals. 1999, New York: McGraw-Hill. 779.

(24) Milazzo, G. Tensioni di vapour di alcune sostanze organiche a bassa temperature. Ann. Chim. 1956, 46: 1105-1111.

(25) Williamham, C.B.; Taylor, W.J.; Pignocco, J.M.; Rossini, F.D. Vapor pressures and boiling points of some paraffin, alkylcyclopentane, alkylcyclohexane, and alkylbenzene hydrocarbons. J. Res. Natl. Bur. Stand. (U.S.) 1945, 35: 219-244.

(26) Downing, R.C. Fluorocarbon refrigerants handbook. 1988, Englewood Cliffs, New Jersey 07632: Prentice-Hall, Inc.

(27) $\mathrm{Bu}, \mathrm{X}$.; Warner, M.J. Solubility of chlorofluorocarbon 113 in water and seawater. Deep sea res. 1995, 42: 1151-1161.

(28) http//www.chemfinder.com

(29) Horvath, A.L.; Getzen, F.W.; Maczynska, Z. J. Phys. Chem. Ref. Data, 1999, 28 (2), 395-627.

(30) Abriola, L.M.; Bradford, S.A. Experimental investigations of the entrapment and persistence of water immiscible contaminants in the subsurface environment. Environ. Health Perspect., 1998, 106, 1083-1095. 
(31) Pankow, J.F.; Cherry, J.A. Dense chlorinated solvents and other DNAPLs in groundwater. Waterloo Press, Portland, Oregon, U.S.A., 1996.

(32) Poulsen, M.M.; Kueper, B.H. A field experiment to study the behaviour of tetrachloroethylene in unsaturated porous media. Environ. Sci. Technol., 1992, 26 (5), 889-895.

(33) Jarsjö, J.; Destouni, G.; Yaron, B. Retention and volatilisation of kerosene: Laboratory studies on glacial and post-glacial soils. J. Contam. Hydrol., 1994, $17,167-185$.

(34) Christophersen, M.; Broholm, M.M.; Mosbæk, H.; Karapanagioti, H.; Burganos, V.N.; Kjeldsen, P. Transport of hydrocarbons from an emplaced fuel source experiment in the vadose zone at Airbase Værløse, Denmark. Accepted for publication in J. Contam. Hydrol., 2005.

(35) Mayer, K.U., Frind, E. O. and Blowes, D.W. Multicomponent reactive transport modeling in variably saturated porous media using a generalized formulation for kinetically controlled reactions. Water Resour. Res., 2002, 38(9), 1174-1195.

(36) Hölting, B. Hydrogeologie. Stuttgart, Enke., 1992, 415 p.

(37) Lange, F. M. Physical properties and estimation of van Genuchten parameters from soil samples of the Airbase Varlфse/DK. 2002. Hohenheim. Survey Report. 